\title{
Parametric Feedback Resonance in Chaotic Systems
}

\author{
H. G. Schuster* and E. Niebur ${ }^{\dagger}$ \\ Computation and Neural Systems Program, California Institute of Technology, \\ Pasadena, California 91125 \\ E. R. Hunt, G. A. Johnson, and M. Löcher \\ Condensed Matter and Surface Science Program and Department of Physics and Astronomy, Ohio University, \\ Athens, Ohio 45701-2979 \\ (Received 11 July 1994; revised manuscript received 31 August 1995)
}

\begin{abstract}
If one changes the control parameter of a chaotic system proportionally to the distance between an arbitrary point on the strange attractor and the actual trajectory, the lifetime $\tau$ of the most stable unstable periodic orbit in the vicinity of this point starts to diverge with a power law. The volume in parameter space where $\tau$ becomes infinite is finite and from its nonfractal boundaries one can determine directly the local Liapunov exponents. The experimental applicability of the method is demonstrated for two coupled diode resonators.
\end{abstract}

PACS numbers: 05.45.+b, 07.50.Ek, 47.20.-k

Unstable periodic orbits are the building blocks of chaos in nonlinear dynamical systems and therefore of fundamental interest in physics [1-3]. Since linear systems resonate under periodic forcing with their building blocks, i.e., harmonic oscillators, we are led naturally to the question which we will address in this Letter: Under which time-dependent perturbation will a strongly chaotic nonlinear system resonate with an unstable periodic orbit, and what can be learned from this resonance?

We start from two recent results. First, it has been shown by Ott, Grebogi, and Yorke [4] that small timedependent changes of the control parameter of a chaotic system could stabilize a prescribed periodic orbit completely such that the system becomes nonchaotic [5]. Second, it has been demonstrated by Hunt [6] that in order to achieve this effect it is generally sufficient to change the control parameter proportionally to the difference between the desired orbit and the trajectory of the system.

Here we build on these observations by investigating the behavior of a chaotic system under parametric feedback. However, we will not focus a priori on a special orbit but show that if we choose the parameter change proportional to the difference between any point on the attractor [7] and the actual trajectory, the system resonates with the most stable unstable periodic orbit close to this point. The resonance shows up as a divergence of the lifetime $\tau$ of this unstable periodic orbit and follows a power law. We will also show that there is even in higher dimensional systems a finite volume in parameter space where $\tau$ becomes infinite, and from its measurable nonfractal boundaries one can determine directly all local Liapunov exponents [8]. The experimental applicability of the method is demonstrated for two coupled diode resonators.

We first consider a one-dimensional map and generalize later to higher dimensional systems. The invariant density $\rho(x)$ can be expanded in terms of periodic orbits [9], $\rho(x)=\sum_{F i x} \delta\left(x-x^{*}\right) \exp \left[-n / \tau\left(x^{*}\right)\right] /$
$\sum_{F i x} \exp \left[-n \tau\left(x^{*}\right)\right]$ where the sums run over all fixed points $x^{*}=f_{r}^{n}\left(x^{*}\right)$ of the $n$-fold iterated map $f$ and the limit $n \rightarrow \infty$ is understood in this equation. The lifetime $\tau$ of a periodic orbit which contains $x^{*}$ is determined by the slopes of the map taken at the orbit points,

$$
\tau^{-1}\left(x^{*}\right)=\frac{1}{n} \log \left|f_{r}^{n^{\prime}}\left(x^{*}\right)\right| .
$$

We now disturb the map locally around a simple fixed point $x_{0}^{*}$ by changing the control parameter $r$ by an amount $\delta r^{t}=\epsilon\left(x_{0}^{*}-x^{t}\right)$ if $\left|x_{0}^{*}-x^{t}\right|<\delta$, with $\delta$ typically of order $10^{-2}$ to $10^{-4}$. After linearizing the equation of motion $x^{t+1}=f_{r}\left(x^{t}\right)$ around $x_{0}^{*}$, we obtain that the slope of the map at $x_{0}^{*}$ changes to $(\partial f / \partial x)\left(x_{0}^{*}\right)-\epsilon(\partial f / \partial r)\left(x_{0}^{*}\right)$ and the lifetime $\tau$, defined by $\tau^{-1}=\log \left|f^{\prime}\left(x^{*}\right)-\epsilon(\partial f / \partial r)\left(x^{*}\right)\right|$, diverges at

$$
\boldsymbol{\epsilon}_{ \pm}=\left[f^{\prime}\left(x_{0}^{*}\right) \pm 1\right] / \frac{\partial f}{\partial r}\left(x_{0}^{*}\right) .
$$

Figure 1 shows $\tau^{-1}(\epsilon)$ for the logistic map [10] $x^{t+1}=$ $r x^{t}\left(1-x^{t}\right)$ at $r=3.79$. One sees that $\tau(\epsilon)$ displays a resonance that starts at $\epsilon=0$ and extends to large values of $\epsilon$. By linearizing around $\epsilon_{ \pm}$, we obtain from Eq. (2) the result that the lifetime $\tau$ diverges proportionally to $\left|\epsilon-\epsilon_{ \pm}\right|^{-1}$ for $\left|\epsilon-\epsilon_{ \pm}\right| \ll 1$. The inset in Fig. 1 confirms this power law for several decades. Equation (2) shows also that the local slope $f^{\prime}\left(x_{0}^{*}\right)$ (i.e., the local Liapunov exponent) and the parameter dependence $(\partial f / \partial r)\left(x_{0}^{*}\right)$ can be obtained directly from the measurable locations of the two critical points $\epsilon_{ \pm}$.

This result can be generalized in a straightforward fashion to periodic orbits of arbitrary order $n$ [11]. It should be clear from these considerations that if we apply parametric feedback which is localized within an interval $2 \delta$ around an arbitrary point $x^{*}$ that that unstable periodic orbit which becomes stabilized first will start to resonate. Its increase of lifetime can be observed in the invariant 


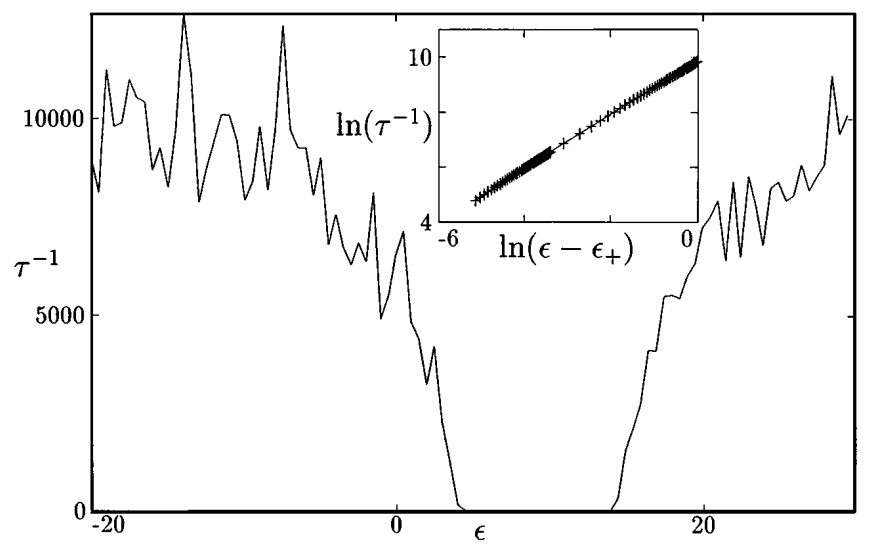

FIG. 1. Stabilization of the unstable fixed point of the logistic map at $r=3.79$, chosen as $(r-1) / r$. Shown is the inverse fraction of time $\tau^{-1}$ that the trajectory spends in the vicinity of the stabilized point, within a distance of $\delta=10^{-4}$. This inverse fraction can vary between unity (if the system stays always within the stabilization interval) and infinity (if it never enters this interval). The figure shows that for $4.67<\epsilon<$ 14.3, this fraction is unity. For $\epsilon=0, \tau^{-1}$ is close to its unperturbed value of $5 \times 10^{3}$. The inset illustrates the power law by showing the inverse time in a log-log plot at the right border of the stabilized region; $\epsilon_{+}=14.3$.

density or in the power spectrum of the system [12]. Figure 2 shows how for the logistic map an orbit of higher order close to an arbitrarily chosen point becomes stabilized, even in the presence of additive noise.

In higher dimensional systems whose dynamics are described by $d$-dimensional maps $\vec{x}^{t+1}=\vec{f}\left(\vec{x}^{t}\right)$, Eq. (1) for the lifetime of a periodic orbit of order $n$ [determined by $\left.\vec{x}^{*}=\vec{f}^{n}\left(\vec{x}^{*}\right)\right]$ generalizes to $[9,13] \tau^{-1}\left(\vec{x}^{*}\right)=$ $\sum_{i}^{\prime}(1 / n) \log \left|\lambda_{i}\left(x^{*}\right)\right|$. The sum $\sum_{i}^{\prime}$ runs only over all the eigenvalues $\lambda_{i}\left(x^{*}\right)$ of the Jacobian $\left(\partial f_{i}^{n} / \partial x_{j}\right)\left(\vec{x}^{*}\right)$ with modulus larger than unity, i.e., the lifetime is determined only by the local stretching rates. Analogously to the one-dimensional case, we consider an unstable

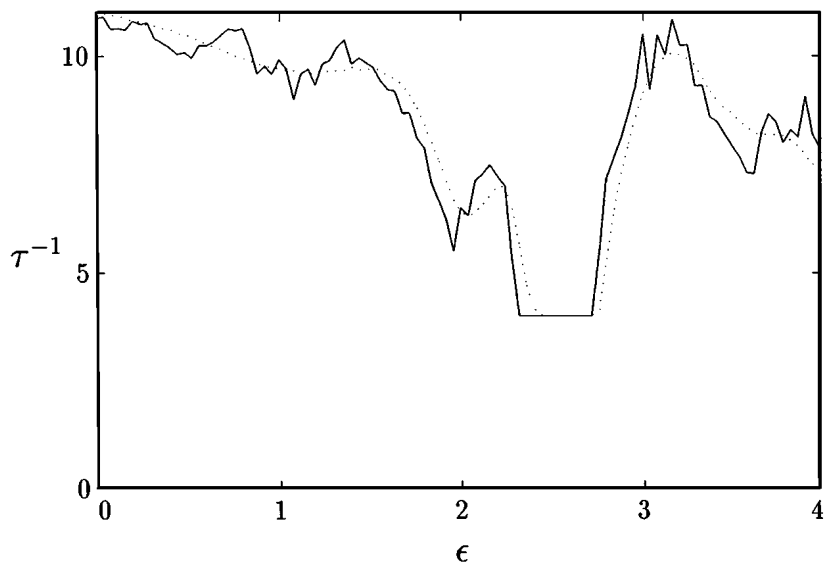

FIG. 2. Stabilization of an arbitrarily chosen orbit of the logistic map at $r=3.79$ around $x^{*}=0.6$ with $\delta=0.03$. Between $\epsilon=2.32$ and $\epsilon=2.76$, an orbit of order 4 is stabilized. The dotted line corresponds to the same values of $x^{*}$ and $\delta$, but random noise with uniform distribution and amplitude $a=10^{-3}$ has been added to the logistic map. fixed point $\vec{x}_{0}^{*}$ and perturb the system by a local parameter change $\delta r^{t}=\vec{\epsilon} \cdot\left(\vec{x}_{0}^{*}-\vec{x}^{t}\right)$ if $\left|\vec{x}^{t}-\vec{x}_{0}^{t}\right| \leq \delta$, where the strengths $\epsilon_{i}(i=1, \ldots, d)$ of the local feedback can be monitored independently [14]. After linearization of the equation of motion $\vec{x}^{t+1}=\vec{f}\left(\vec{x}^{t}\right)$ around $\vec{x}_{0}^{*}$, we obtain that the inverse lifetime of $\vec{x}_{0}^{*}$ changes to $\tau\left(\vec{x}_{0}^{*}\right)^{-1}=\sum_{i}^{\prime}(1 / n) \log \left|\gamma_{i}\left(\vec{x}_{0}^{*}\right)\right|$. Here $\sum_{i}^{\prime}$ runs again only over those $\gamma_{i}$ with modulus larger than unity, and $\gamma_{i}$ are the roots of

$$
\begin{aligned}
\operatorname{det}\{ & \left.\delta_{i j}\left[\lambda_{i}\left(x_{0}^{*}\right)-\gamma\right]-\alpha_{i j}\right\} \\
& =\prod_{i=1}^{d}\left[\lambda_{i}\left(\vec{x}_{0}^{*}\right)-\gamma\right]-\sum_{j} \alpha_{j j} \prod_{i \neq j}\left[\lambda_{i}\left(\vec{x}_{0}^{*}\right)-\gamma\right]=0 .
\end{aligned}
$$

The coefficients $\alpha_{i j}$ are given by $\alpha_{i j}=\vec{e}_{i}^{\ell} \cdot(\partial \vec{f} / \partial r) \times$ $\left(x_{0}^{*}\right)\left(\vec{\epsilon} \cdot \vec{e}_{j}^{r}\right)$ where $\vec{e}_{i}^{\ell}$ and $\vec{e}_{j}^{r}$ are the left and right eigenvectors of the Jacobian $\left(\partial f_{i} / \partial x_{j}\right)\left(x_{0}^{*}\right)$ which belong to the eigenvalues $\lambda_{i}\left(\vec{x}_{0}^{*}\right)$ and $\lambda_{j}\left(\vec{x}_{0}^{*}\right)$. Since $\alpha_{i j}$ is a product of two factors which depend individually only on $i$ or $j$, the determinant in Eq. (3) depends only linearly on $\alpha_{j j}$. We will now exploit this fact to determine the region in parameter space ( $\epsilon$ space) where $\tau$ diverges.

It follows from $\tau^{-1}=\sum_{i}^{\prime}(1 / n) \log \left|\gamma_{i}\right|=(1 / n) \times$ $\log \prod_{i}^{\prime}\left|\gamma_{i}\right|$ that $\tau$ diverges within a hypercube $\left|\gamma_{i}\right| \leq 1$, for all $i$, in $\gamma_{i}$ space. For given $\gamma_{i}$, Eq. (3) becomes a linear equation for the coefficients $\alpha_{j j}$. Since an interchange of the $\gamma_{i}$ 's merely leads to an interchange of the equations for the $\alpha_{j j}$ 's, i.e., to the same $\alpha_{j j}$ 's, it follows that the hypercube in $\gamma_{i}$ space is mapped onto a volume in $\alpha_{j j}$ space which has $d+1$ corners (i.e., a triangle in two dimensions) and which is bounded by $d$ hyperplanes and one curved surface (see Fig. 3). Since the $\epsilon_{i}$ 's follow from the $\alpha_{j j}$ by a linear transformation [15], the volume in $\vec{\epsilon}$ space where $\tau$ diverges is a linearly deformed version of the volume in $\vec{\alpha}$ space. This means that the volume in parameter space for which the lifetime of the unstable fixed point diverges is finite and from its $d+1$ corners one can determine all Liapunov exponents
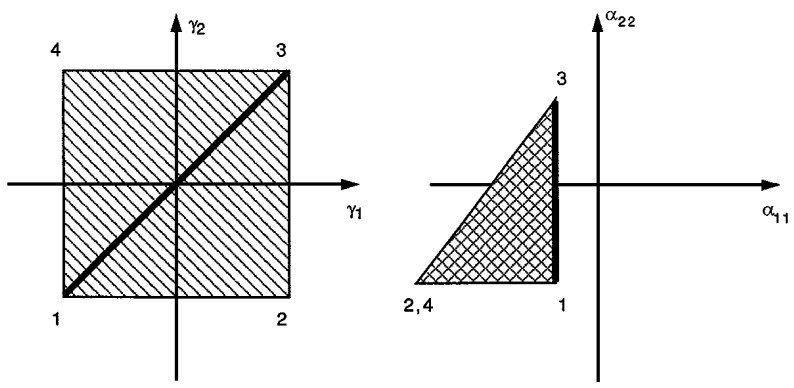

FIG. 3. Schematic representation of the mapping from the space of the eigenvalues $\gamma_{i}$ to the coefficients $\alpha_{j j}$; see Eq. (3). The hypercube in $\gamma$ space (i.e., a square for the twodimensional case shown) is mapped into a "half-hypercube" (a triangle in two dimensions) in $\alpha$ space. This is illustrated by numbering the corners of the square in $\gamma$ space (from 1 to 4 ), and by labeling the corresponding corners in $\alpha$ space by the same numbers. The line $1-3$ (diagonal; thick line) is mapped to one side of the triangle (thick line). 


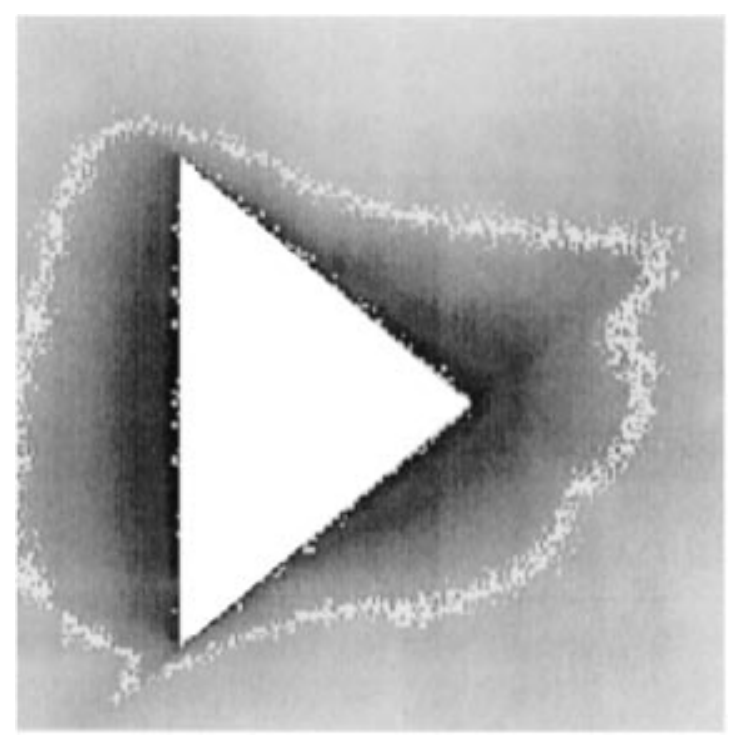

FIG. 4. Stabilization of an unstable orbit of the Hénon map, ( $r=1.4)$, in $\vec{\epsilon}$ space, with $\epsilon_{1}$ varying from -1 (bottom) to 5 (top), and $\epsilon_{2}$ varying from -2.5 (left) to 2.5 (right). The gray level of each pixel represents the inverse fraction $\tau^{-1}$ of the time the trajectory spends in the vicinity $\left(\delta=10^{-3}\right)$ of the fixed point $\left\{x^{*}=\frac{1}{2}\left[-.7+\sqrt{(0.7)^{2}+4 r}\right], y^{*}=x^{*}\right\}$, ranging from black if the inverse fraction is just larger than unity to light gray for the maximal value found in this simulation $(2.6 \times$ $\left.10^{5}\right)$. In the unperturbed system $(\vec{\epsilon}=0), \tau^{-1}=4.3 \times 10^{5}$. In two regions we deviated from this grayscale coding: First, we show in solid white the triangle in which the trajectory never leaves the interval around the unstable fixed point (i.e., $\tau=1.0$ ). Second, we highlight in white the contour on which $\tau$ assumes a value identical $( \pm 5 \%)$ to that found at $\vec{\epsilon}=0$.

$\lambda_{i}$ and the $d \times d$ coefficients of the transformation matrix between $\alpha_{j j}$ and $\epsilon_{i}$ [16].

As an example we consider the Hénon map [10], $x^{t+1}=$ $r+0.3 y^{t}-\left(x^{t}\right)^{2}, y^{t+1}=x^{t}$. Figure 4 shows the triangle in parameter space where the lifetime of the fixed

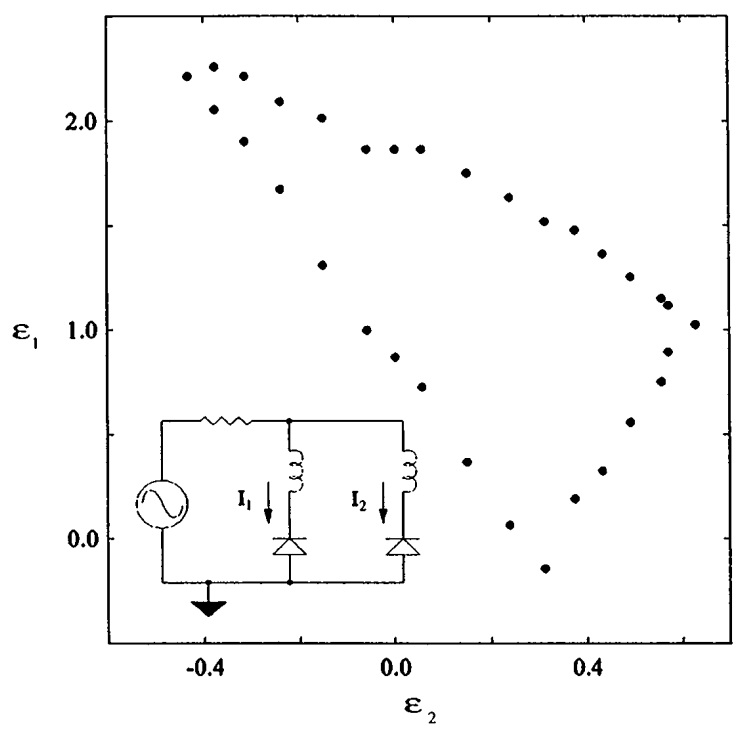

FIG. 5. Region in which control is maintained. The two controllers' gains $\epsilon_{1}$ and $\epsilon_{2}$ are in arbitrary units. The inset shows the electronic circuit used. point $x_{0}^{*}$ diverges. From the corners which correspond to the values $\left[\alpha_{11}, \alpha_{12}\right]=\left[\left(\lambda_{1}^{2}-1\right) /\left(\lambda_{2}-\lambda_{1}\right),\left(\lambda_{2}^{2}-\right.\right.$ 1) $\left./\left(\lambda_{1}-\lambda_{2}\right)\right] ;\left[\left(\lambda_{1}-1\right)^{2} /\left(\lambda_{2}-\lambda_{1}\right),\left(\lambda_{2}-1\right)^{2} /\left(\lambda_{1}-\right.\right.$ $\left.\left.\lambda_{2}\right)\right] ;\left[\left(\lambda_{1}+1\right)^{2} /\left(\lambda_{2}-\lambda_{1}\right),\left(\lambda_{2}+1\right)^{2} /\left(\lambda_{1}-\lambda_{2}\right)\right]$ and from the linear transformation [15] we obtain $\lambda_{1}=-1.92$ and $\lambda_{2}=0.16$ in agreement with a direct computation from $\left(\partial f_{i} / \partial x_{j}\right)\left(\vec{x}_{0}^{*}\right)$.

In order to examine the application of the method experimentally, we choose to employ two coupled diode resonators. This system exhibits the quasiperiod route to chaos and has been previously studied in detail [17]. Further, it has been controlled in a number of low- and high-period orbits [18].

The system, shown in the inset of Fig. 5, is composed of a parallel combination of two diode resonators in series with a resistor, which allows for coupling, and is driven sinusoidally. Each diode resonator is composed of a diode and an inductor. As the drive voltage is increased, the system period doubles once and then undergoes a Hopf bifurcation into a quasiperiodic state. This gives way to mode-locked states, sometimes a second Hopf bifurcation, and chaos. To view the dynamics, we electronically produce a display of its Poincaré section, by sampling and displaying the currents through the two branches of each cycle. The section, following the Hopf bifurcation, appears as two circles, the points of which smoothly rotate in the quasiperiodic region and become stationary in the phase-locked region. With increasing drive the circles break up and the motion becomes chaotic.

The control method used here is described in Ref. [6]. In this method, deviations from a set point, within a window, are fed back to modulate a parameter of the system. For the purpose of this work, we use two controllers, one for each branch, and sum their outputs. Since the system has already period doubled, we sample the currents

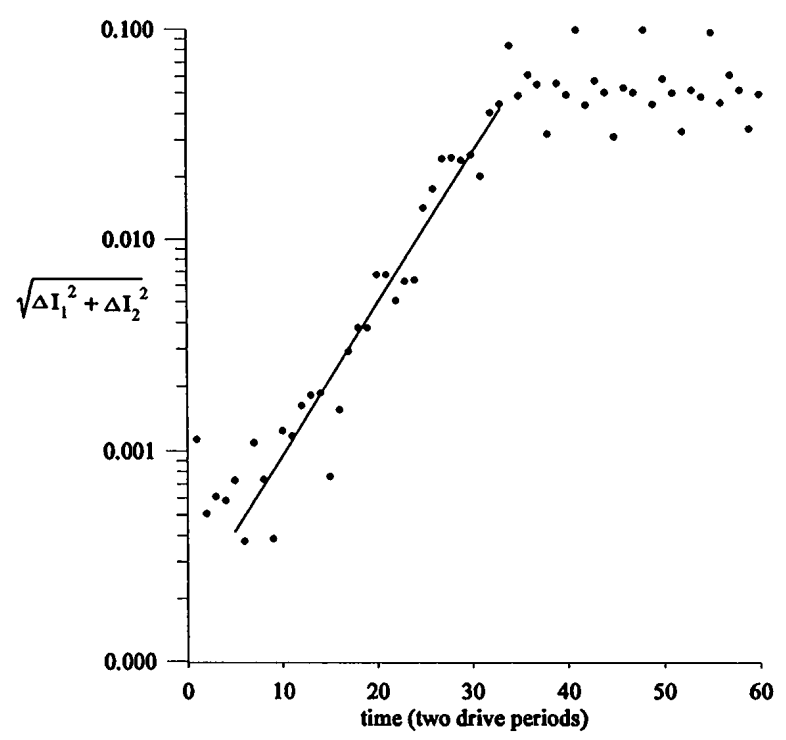

FIG. 6. Time dependence of the magnitude of the deviation of the currents from their value at the fixed point. The line has a slope of 0.16 per two drive periods. 
in the two branches every other drive cycle and apply the feedback signal for two drive cycles. The drive voltage is arbitrarily chosen so the system is at the onset of a phaselocked state. Initially the system is controlled to the preHopf bifurcation state (still period 2) and the set points are adjusted to the fixed points by minimizing the feedback signal. The gain of each controller $\epsilon_{1}$ and $\epsilon_{2}$ may then be adjusted to determine the range over which control is maintained.

The magnitude of the complex local Liapunov exponent can be determined by the rate at which the orbit spirals away from the fixed point. Figure 6 shows the deviation of the currents after control on the fixed point has been switched off. We find for the slope $0.16 \pm 0.02$ per two drive periods, which gives $10^{0.16}=1.45$ for $\operatorname{Re}(\lambda)$. The phase angle can be obtained from the winding number, which for our phase-locked state is $\frac{3}{14}$. This gives an angle of $\operatorname{Im}(\lambda)=2 \pi \times \frac{3}{7}$ using only one of the circles. The ratio $\operatorname{Im} \lambda / \operatorname{Re} \lambda=7.3$ agrees with the value obtained from the corners 7.1 [16].

Since the conditions for the occurrence of parametric feedback resonance are rather weak [19] it should be observable in many systems, offering experimenters a fast analog method to study unstable periodic orbits and the corresponding local Liapunov exponents [20].

This work was supported by NATO Grant No. CRG 911034, NSF Grant No. BIR 92-14238, and ONR Grant No. N0014-94-10395. We gratefully acknowledge discussions with R. W. Rollins and D. Cigna. H. G. S. thanks C. Koch for the kind hospitality extended to him during his stay at Caltech.

*Permanent address: Institut für Theoretische Physik, Universität Kiel, Olshausenstrasse 40, D-24098 Kiel 1, Germany.

${ }^{\dagger}$ To whom correspondence should be addressed. Present address: Krieger Mind/Brain Institute and Department of Neuroscience, The John Hopkins University, 3400 N. Charles Street, Baltimore, MD 21218.

[1] H. Poincaré, Les Méthodes Nouvelles de la Mécanique Céleste (Gauthiers Villers, Paris, 1892).

[2] D. Ruelle, Statistical Mechanics, Thermodynamic Formalism (Addison-Wesley, Reading, MA, 1978).

[3] R. Artuso, E. Aurell, and P. Cvitanović, Nonlinearity 3, 325 (1990).

[4] E. Ott, C. Grebogi, and J. A. Yorke, Phys. Rev. Lett. 64, 1196 (1990).

[5] T. Shinbrot, C. Grebogi, E. Ott, and J. A. Yorke, Nature (London) 363, 411 (1993).

[6] E. R. Hunt, Phys. Rev. Lett. 67, 1953 (1991).

[7] The strange attractor is the closure of all unstable periodic orbits of the dynamical system [9]. Therefore, every point on the attractor is arbitrarily close to a periodic orbit, i.e., to fixed points of the $n$-fold iterated map.

[8] The local Liapunov exponents determine the local stability of an unstable periodic orbit, in contrast to the global Liapunov exponents which follow from averaging over the whole attractor.
[9] G. Gunaratne and I. Procaccia, Phys. Rev. Lett. 59, 1377 (1987).

[10] H. G. Schuster, Deterministic Chaos (VCH Publishers, Weinheim, 1989), 2nd ed.

[11] For a periodic cycle with points $x_{1}^{*}, \ldots, x_{n}^{*}$ and inverse lifetime $\tau^{-1}=(1 / n) \log \left|f^{n^{\prime}}\left(x_{i}^{*}\right)\right|$, a parameter change $\delta r^{t}=\epsilon\left(x_{i}^{*}-x^{t}\right)$ for $\left|x_{i}^{*}-x^{t}\right|<\delta$ (where $1 \leq i \leq n$ is arbitrary but fixed) changes $\tau$ to $\tau^{-1}=(1 / n) \log \left|f^{n^{\prime}}\left(x_{i}^{*}\right)-\epsilon\left(\partial f^{n} / \partial r\right)\left(x_{i}^{*}\right)\right|$ which diverges at $\epsilon_{ \pm}=\left[f^{n^{\prime}}\left(x_{i}^{*}\right) \pm 1\right] /\left(\partial f^{n} / \partial r\right)\left(x_{i}^{*}\right)$. This result holds if $\delta$ is small enough, i.e., $\delta<\min _{i \neq j}\left|x_{i}^{*}-x_{j}^{*}\right| / 2$ such that $\delta r^{t}$ affects only a single point on the cycle.

[12] F. Christiansen, G. Paladin, and H. H. Rugh, Phys. Rev. Lett. 65, 2087 (1990), and references therein.

[13] L. P. Kadanoff and C. Tang, Proc. Natl. Acad. Sci. U.S.A. 81, 1276 (1984).

[14] The $\epsilon_{i}$ are the components of a vector in a space dual to phase space. We will call this the "parameter space."

[15] $\alpha_{j j}=\sum_{m}\left[\vec{e}_{j}^{\ell} \cdot(\partial \vec{f} / \partial r)\left(x_{0}^{*}\right)\right] \vec{e}_{j}^{r}(m) \epsilon_{m}$.

[16] If the eigenvalues $\left\{\lambda_{i}\right\}$ of $\left(\partial f_{i} / \partial x_{j}\right)\left(x_{0}^{*}\right)$ are complex, the equations for the real control parameters become in two dimensions $\operatorname{Re} \alpha_{11}=\sum_{m} \operatorname{Re}\left[\vec{e}^{\ell}(\partial \vec{f} / \partial r)\left(x_{0}^{*}\right) \vec{e}^{r}(m)\right] \epsilon_{m}$, $\operatorname{Im} \alpha_{11}=\sum_{m} \operatorname{Re}\left[\vec{e}^{\ell}(\partial \vec{f} / \partial r)\left(x_{0}^{*}\right) \vec{e}^{r}(m)\right] \epsilon_{m}$. In terms of the corners $\left(\epsilon_{x}^{1}, \epsilon_{y}^{1}\right),\left(\epsilon_{x}^{2}, \epsilon_{y}^{2}\right),\left(\epsilon_{x}^{3}, \epsilon_{y}^{3}\right)$ in parameter space which correspond to $\left[\gamma_{1}, \gamma_{2}\right]=[1,1] ;[-1,-1] ;[1,-1]$, the matrix $(a, b ; c, d)$ in $\operatorname{Re} \alpha_{11}=a \epsilon_{x}+b \epsilon_{y}$, $\operatorname{Im} \alpha_{11}=c \epsilon_{x}+d \epsilon_{y}$ and the real and imaginary parts of the local Liapunov exponents $\operatorname{Re} \lambda$ and $\operatorname{Im} \lambda$ become

$$
\begin{aligned}
a= & -\left[2\left(\epsilon_{y}^{2}-\epsilon_{y}^{3}\right)+\epsilon_{y}^{1}-\epsilon_{y}^{2}\right] \\
& /\left[\left(\epsilon_{x}^{1}-\epsilon_{x}^{2}\right)\left(\epsilon_{y}^{2}-\epsilon_{y}^{3}\right)-\left(\epsilon_{x}^{2}-\epsilon_{x}^{3}\right)\left(\epsilon_{y}^{1}-\epsilon_{y}^{2}\right)\right], \\
b= & {\left[2\left(\epsilon_{x}^{2}-\epsilon_{x}^{3}\right)+\epsilon_{x}^{1}-\epsilon_{x}^{2}\right] } \\
& /\left[\left(\epsilon_{x}^{1}-\epsilon_{x}^{2}\right)\left(\epsilon_{y}^{2}-\epsilon_{y}^{3}\right)-\left(\epsilon_{x}^{2}-\epsilon_{x}^{3}\right)\left(\epsilon_{y}^{1}-\epsilon_{y}^{2}\right)\right], \\
\operatorname{Re} \lambda= & \left(a \epsilon_{x}^{3}+b \epsilon_{y}^{3}\right), \\
h= & -\left[\left(\epsilon_{x}^{1}-\epsilon_{x}^{2}\right)-\operatorname{Re} \lambda\left(\epsilon_{x}^{1}+\epsilon_{x}^{2}-2 \epsilon_{x}^{3}\right)\right] \\
& /\left[\left(\epsilon_{y}^{1}-\epsilon_{y}^{2}\right)-\operatorname{Re} \lambda\left(\epsilon_{y}^{1}+\epsilon_{y}^{2}-2 \epsilon_{y}^{3}\right)\right] \\
\operatorname{Im} \lambda= & \pm\left\{(\operatorname{Re} \lambda)^{2}-1+4 \operatorname{Re} \lambda\left(\epsilon_{x}^{3}+h \epsilon_{y}^{3}\right)\right. \\
& \left./\left[\epsilon_{x}^{1}-\epsilon_{x}^{2}+h\left(\epsilon_{y}^{1}-h \epsilon_{y}^{2}\right)\right]\right\}^{1 / 2} .
\end{aligned}
$$

Note that the Liapunov exponents depend only on the ratios of the control parameters.

[17] Z. Su, R. W. Rollins, and E. R. Hunt, Phys. Rev. A 40, 2689-2698 (1989).

[18] E. R. Hunt and G. A. Johnson, in Proceedings of the 2nd Experimental Chaos Conference, edited by W. Ditto (World Scientific, Singapore, 1995), p. 140.

[19] Even in high-dimensional systems, resonances can be generated by varying only one control parameter $r$, provided that its change affects all directions, i.e., if $\vec{e}_{i}^{\ell} \cdot(\partial / \partial r) \vec{f}\left(x^{*}\right) \neq 0$ for all $i$.

[20] If we linearize the equations of motion $x^{t+1}=f\left(x^{t}\right)$ for $\left|x^{*}-x^{t}\right|<\delta$ around the fixed point $x_{0}^{*}$ and call $\lambda=$ $\left|(\partial f / \partial r)\left(x^{*}\right)\right|$, the distribution of lifetimes $P(\tau)$ becomes $P(\tau) \propto \delta e^{-\tau \log \lambda} \rho\left[x^{*}+\delta e^{-\epsilon \log \lambda}\right]$, where $\rho(x)$ is the invariant density with which points are injected into the interval $\left|x^{*}-x\right|<\delta$. 